\title{
Exponential stabilization by delay feedback control for highly nonlinear hybrid stochastic functional differential equations with infinite delay
}

\author{
Chunhui Mei ${ }^{\mathrm{a}, \mathrm{b}}$, Chen $\mathrm{Fei}^{\mathrm{c}, \mathrm{d}}$, Weiyin Fei ${ }^{\mathrm{b}, *}$, Xuerong Mao ${ }^{\mathrm{e}}$ \\ ${ }^{a}$ School of Science, Nanjing University of Science and Technology, Nanjing, Jiangsu 210094, China \\ ${ }^{b}$ The Key Laboratory of Advanced Perception and Intelligent Control of High-end Equipment, Ministry of Education, and School of \\ Mathematics-Physics and Finance, Anhui Polytechnic University, Wuhu, 241000, China \\ ${ }^{c}$ Business School, University of Shanghai for Science and Technology, Shanghai, 200093, China \\ ${ }^{d}$ Glorious Sun School of Business and Management, Donghua University, Shanghai, 200051, China \\ ${ }^{e}$ Department of Mathematics and Statistics, University of Strathclyde, Glasgow G1 1XH, UK
}

\begin{abstract}
Given an unstable hybrid stochastic functional differential equation, how to design a delay feedback controller to make it stable? Some results have been obtained for hybrid systems with finite delay. However, the state of many stochastic differential equations are related to the whole history of the system, so it is necessary to discuss the feedback control of stochastic functional differential equations with infinite delay. On the other hand, in many practical stochastic models, the coefficients of these systems do not satisfy the linear growth condition, but are highly nonlinear. In this paper, the delay feedback controls are designed for a class of infinite delay stochastic systems with highly nonlinear and the influence of switching state.
\end{abstract}

Keywords: Infinite delay; Markovian switching; M-matrix; Highly nonlinear; Delay feedback control

\section{Introduction}

In many engineering and science field, due to many systems are affected by time delay and random factors, we often use stochastic functional differential equations (SFDEs in short) or stochastic delay differential equations (SDDEs in short) to describe such systems. Recently, theories of SFDEs including stability and their applications have attracted much of researchers' attention ( see, e.g., $[1,2,3,4,5])$. Furthermore, in many practical problems, the current state of the system may be related to all the previous history, so many scholars use the stochastic functional differential equations with infinite delay (ISFDEs in short) to model these systems and study their various properties (see, e.g., $[6,7,8,9,10]$ ).

The previous results on stability generally require that the coefficients of stochastic systems satisfy both the local Lipschitz condition and the linear growth condition. However, in many ecological and economic models, the coefficients of the system may not meet the linear growth conditions, but have highly nonlinear characteristics $([11,12,13,14,15,16])$. Therefore, Hu et al. [17] further considered the exponential stability and robustness of a class of ISFDEs which do not satisfy the linear growth condition. Wu and $\mathrm{Hu}$ [18] extended the stochastic version of LaSalle theorem established by [19] to the infinite delay, and discussed the attraction, stability and robustness of ISFDEs.

On the other hand, continuous-time Markov chains are often used to model the system whose structures and parameters may be abrupt changes. Hence, SFDEs with Markovian switching, known also as hybrid SFDEs, have appeared frequently in practice. The stability is a fundamental problem in the study of hybrid SFDEs (see e.g. [20, $21,22,23,24])$. Correspondingly, the control and stabilization of hybrid stochastic systems have been received the

\footnotetext{
${ }^{*}$ Corresponding author

Email addresses: mch413@163.com (Chunhui Mei), jasmine9366@163.com (Chen Fei), wyfei@ahpu.edu.cn (Weiyin Fei), x.mao@strath.ac.uk (Xuerong Mao)
} 
increasing attentions (see e.g.[25, 26, 27, 28, 29, 30, 31]). Mao [32] proposed for the first time that a delay feedback controller can be designed to stabilize an unstable hybrid stochastic differential equation. Lu et al. [33] studied how to use the delay feedback control to make the unstable highly nonlinear hybrid stochastic differential equation asymptotically stable, and obtained an upper bound of time lag. Using the method of M-matrix, for hybrid SDDEs, Li and Mao [34] designed delay feedback control to make the controlled system not only asymptotically stable in the sense of moment, but also to guarantee the exponential stability in the sense of moment and almost surely. In this paper, we will further extend the above results to hybrid infinite delay systems. Comparing with the existing papers, we highlight a number of main contributions of this paper:

(i) As mentioned before, systems with infinite delay often appear in population models, and such biological models often do not satisfy the linear growth conditions. The main purpose of this paper is to design a class of delay feedback controllers to stabilize unstable hybrid ISFDEs. In order to overcome the difficulties caused by infinite delay and highly nonlinear, the exponential stability of the controlled ISFDEs is obtained by reasonably selecting the phase space, constructing the appropriate probability measure space, and ensuring the asymptotic boundedness of the system.

(ii) In this paper, the phase space is $B C\left((-\infty, 0] ; R^{n}\right)$. After choosing this phase space, we not only generalize the results of hybrid SDDEs in [34] to the functional systems with infinite delay, but also improve the results of theorem 4.4 and 4.5 in [34]. That is, we get a better upper bound of time delay to reduce the conservatism.

(iii) Different from the previous stability results of ISFDEs, considering the influence of Markovian switching on the system, we mainly use the M-matrix method, which makes our results not only related to the coefficients of the subsystems for better verification, but also take into account the influence of different modes.

\section{Notations and Preliminaries}

Throughout this paper, unless otherwise specified, we use the following notations. Let $R_{+}=[0, \infty)$. If both $a, b$ are real numbers, then $a \wedge b=\min \{a, b\}$ and $a \vee b=\max \{a, b\}$. For $x \in R^{n},|x|$ denotes its Euclidean norm. If $A$ is a vector or matrix, its transpose is denoted by $A^{T}$. For $A \in R^{n \times m}$, we let $|A|=\sqrt{\operatorname{trace}\left(A^{T} A\right)}$ be its Frobenius norm. If $A$ is a symmetric real-valued matrix $\left(A=A^{T}\right)$, denote by $\lambda_{\max }(A)$ and $\lambda_{\min }(A)$ its largest and smallest eigenvalue, respectively. By $A \leq 0$ and $A<0$, we mean $A$ is non-positive and negative definite, respectively.

Let $\left(\Omega, \mathcal{G},\left\{\mathcal{G}_{t}\right\}_{t \geq 0}, \mathbb{P}\right)$ be a complete probability space with a natural filtration $\left\{\mathcal{G}_{t}\right\}_{t \geq 0}$ satisfying the usual conditions (i.e., it is increasing and right continuous while $\mathcal{G}_{0}$ contains all $\mathbb{P}$-null sets). If $G$ is a subset of $\Omega$, denote by $I_{G}$ its indicator function; that is, $I_{G}(\omega)=1$ if $\omega \in G$ and 0 otherwise. Let $w(t)=\left(w_{1}(t), \cdots, w_{m}(t)\right)^{T}$ be an $m$-dimensional Brownian motion defined on the probability space. Let $r(t), t \geq 0$, be a right-continuous Markov chain on the probability space taking values in a finite state space $\mathcal{S}=\{1,2, \cdots, N\}$ with generator $\Gamma=\left(\gamma_{i j}\right)_{N \times N}$ given by

$$
\mathbb{P}\{r(t+\Delta)=j \mid r(t)=i\}= \begin{cases}\gamma_{i j} \Delta+o(\Delta) & \text { if } i \neq j, \\ 1+\gamma_{i i} \Delta+o(\Delta) & \text { if } i=j,\end{cases}
$$

where $\Delta>0$. Here $\gamma_{i j} \geq 0$ is the transition rate from $i$ to $j$ if $i \neq j$ while

$$
\gamma_{i i}=-\sum_{j \neq i} \gamma_{i j}
$$

We always assume that the Markov chain $r(\cdot)$ is independent of the Brownian motion $w(\cdot)$.

Denote by $C\left((-\infty, 0] ; R^{n}\right)$ the family of continuous functions $\varphi$ from $(-\infty, 0] \rightarrow R^{n}$. Similarly, denote by $B C\left((-\infty, 0] ; R^{n}\right)$ the family of bounded continuous functions $\varphi$ from $(-\infty, 0] \rightarrow R^{n}$ with the norm $\|\varphi\|=\sup _{s \leq 0}|\varphi(s)|$. If $x(t)$ is an $R^{n}$-valued stochastic process, we let $x_{t}=x_{t}(s)=\{x(t+s):-\infty<s \leq 0\}$ for $t \geq 0$. Let $L^{r}\left((-\infty, 0] ; R^{n}\right)$ denote all functions $h:(-\infty, 0] \rightarrow R^{n}$ such that $\int_{-\infty}^{0}|h(s)|^{r} d s<\infty$. We give the following lemma, whose proof is standard.

Lemma 2.1. Let $\varphi \in B C\left((-\infty, 0] ; R^{n}\right) \cap L^{r}\left((-\infty, 0] ; R^{n}\right)$ for any $r>0$. Then for all $r_{1}>r, \varphi \in B C\left((-\infty, 0] ; R^{n}\right) \cap$ $L^{r_{1}}\left((-\infty, 0] ; R^{n}\right)$. 
Let $\mathcal{P}_{0}$ denote all probability measures $\mu$ on $(-\infty, 0]$. For each $\varepsilon>0$, define

$$
\mathcal{P}_{\varepsilon}=\left\{\mu \in \mathcal{P}_{0} ; \mu^{(\varepsilon)}:=\int_{-\infty}^{0} e^{-\varepsilon \theta} d \mu(\theta)<\infty\right\} .
$$

In fact, there are many probability measures that meet the above requirements. Here are just two examples we will use.

(i) Fix $\tau>0$, let $v$ be the Dirac measure at $-\tau$ ( see, e.g. [35, p.9],). Then for any $\varepsilon>0$,

$$
v^{(\varepsilon)}:=\int_{-\infty}^{0} e^{-\varepsilon \theta} d v(\theta)=e^{\varepsilon \tau}<\infty,
$$

which means $v \in \mathcal{P}_{\varepsilon}$.

(ii) Let $d \mu(\theta)=\varepsilon_{0} e^{\varepsilon_{0} \theta} d \theta$. Then, $\mu \in \mathcal{P}_{0}$ and for any $\varepsilon \in\left(0, \varepsilon_{0}\right)$,

$$
\mu^{(\varepsilon)}:=\varepsilon_{0} \int_{-\infty}^{0} e^{\left(\varepsilon_{0}-\varepsilon\right) \theta} d \theta=\frac{\varepsilon_{0}}{\varepsilon_{0}-\varepsilon}<\infty
$$

which also means $\mu \in \mathcal{P}_{\varepsilon}$ for $\forall \varepsilon \in\left(0, \varepsilon_{0}\right) . \mu^{(\varepsilon)}$ has the following nice property. We give it as a lemma.

Lemma 2.2. (cf. [17]) Fix $\varepsilon_{1}>0$. For any $\varepsilon_{1}>\varepsilon>0, \mu^{(\varepsilon)}$ is continuously nondecreasing on $\varepsilon$ and satisfies $\mu^{\left(\varepsilon_{1}\right)}>\mu^{(\varepsilon)}>\mu^{(0)}=1$. Meanwhile ,we have $\mathcal{P}_{0} \supset \mathcal{P}_{\varepsilon} \supset \mathcal{P}_{\varepsilon_{1}}$.

Let

$$
f: B C\left((-\infty, 0] ; R^{n}\right) \times \mathcal{S} \times R_{+} \rightarrow R^{n} \text { and } g: B C\left((-\infty, 0] ; R^{n}\right) \times \mathcal{S} \times R_{+} \rightarrow R^{n \times m} .
$$

be both Borel measurable functions. Consider a nonlinear hybrid stochastic functional differential equation

$$
d x(t)=f\left(x_{t}, r(t), t\right) d t+g\left(x_{t}, r(t), t\right) d w(t), t \geq 0
$$

with the initial data $\{\xi(\theta):-\infty<\theta \leq 0\}=\xi \in B C\left((-\infty, 0] ; R^{n}\right)$ and $i_{0} \in \mathcal{S}$, where $x_{t}$ is a $B C\left((-\infty, 0] ; R^{n}\right)$-valued stochastic process.

As a standing hypothesis, we assume the the coefficient $f$ or $g$ are local lipschtiz continuous and polynomial growth condition in this paper. For this reason, we give the following hypothesis.

Assumption 2.3. For each real number $b>0$, there is a constant $K_{b}>0$ such that

$$
|f(\varphi, i, t)-f(\phi, i, t)| \vee|g(\varphi, i, t)-g(\phi, i, t)| \leq K_{b}\|\varphi-\phi\|
$$

for all $\varphi, \phi \in B C\left((-\infty, 0] ; R^{n}\right)$ with $\|\varphi\| \vee\|\phi\| \leq b$ and all $(i, t) \in \mathcal{S} \times R_{+}$. Moreover, there are three constants $K>0$, $q_{1} \geq 1$ and $q_{2} \geq 1$ as well as two probability measures $\mu_{1}, \mu_{2}$ on $(-\infty, 0]$ such that

$$
\begin{array}{r}
|f(\varphi, i, t)| \leq K\left(\int_{-\infty}^{0}|\varphi(\theta)|^{q_{1}} d \mu_{1}(\theta)+|\varphi(0)|^{q_{1}}+\int_{-\infty}^{0}|\varphi(\theta)| d \mu_{1}(\theta)+|\varphi(0)|\right) \\
\text { and }|g(\varphi, i, t)| \leq K\left(\int_{-\infty}^{0}|\varphi(\theta)|^{q_{2}} d \mu_{2}(\theta)+|\varphi(0)|^{q_{2}}+\int_{-\infty}^{0}|\varphi(\theta)| d \mu_{2}(\theta)+|\varphi(0)|\right)
\end{array}
$$

for all $(\varphi, i, t) \in B C\left((-\infty, 0] ; R^{n}\right) \times \mathcal{S} \times R_{+}$.

Obviously, only under the condition of Assumption 2.3, system (2.4) may explode in a finite time. In this paper, we give a criterion for the existence and uniqueness of solutions of stochastic functional equations by using Lyapunov function (also known as generalized Khasminskiis condition). 
Assumption 2.4. Let $q_{1}, q_{2}, \mu_{1}, \mu_{2}$ be the same as in Assumption 2.3. Assume that there are some positive constants $q, p, \alpha_{k}, \beta_{k}(k=1,2,3)$ such that

$$
q \geq\left(2 q_{1}\right) \vee\left(q_{1}+2 q_{2}-1\right), p \geq\left(q_{1}+1\right) \vee\left(2 q_{2}\right) \text { and } \alpha_{3}>\alpha_{1}+\alpha_{2}
$$

while

$$
\begin{aligned}
\varphi(0)^{T} f(\varphi, i, t)+\frac{q-1}{2}|g(\varphi, i, t)|^{2} \leq & \sum_{k=1}^{2} \alpha_{k} \int_{-\infty}^{0}|\varphi(\theta)|^{p} d \mu_{k}(\theta)-\alpha_{3}|\varphi(0)|^{p} \\
& +\sum_{k=1}^{2} \beta_{k} \int_{-\infty}^{0}|\varphi(\theta)|^{2} d \mu_{k}(\theta)+\beta_{3}|\varphi(0)|^{2}
\end{aligned}
$$

for all $(\varphi, i, t) \in B C\left((-\infty, 0] ; R^{n}\right) \times \mathcal{S} \times R_{+}$.

Next, as long as we limit the initial value of the equation, we can give the existence and uniqueness of the solution of the stochastic system.

Theorem 2.5. Under Assumptions 2.3, 2.4. The equation (2.4) has a unique global solution $x(t)$ on $t \in R$, for any given initial data

$$
\xi \in B C\left((-\infty, 0] ; R^{n}\right) \cap L^{2}\left((-\infty, 0] ; R^{n}\right) \text { and } i_{0} \in \mathcal{S}
$$

Proof. We divide the proof into two steps.

Step 1. Let $V(x)=|x|^{q}$. We define a functional $\mathcal{L}_{1} V: B C\left((-\infty, 0] ; R^{n}\right) \times \mathcal{S} \times R_{+} \rightarrow R$ by

$$
\begin{aligned}
\mathcal{L}_{1} V(\varphi, i, t)= & q|\varphi(0)|^{q-2} \varphi(0)^{T} f(\varphi, i, t)+\frac{q}{2}|\varphi(0)|^{q-2}|g(\varphi, i, t)|^{2} \\
& +\frac{q(q-2)}{2}|\varphi(0)|^{q-4}\left|\varphi(0)^{T} g(\varphi, i, t)\right|^{2} .
\end{aligned}
$$

By Assumptions 2.4, we then derive

$$
\begin{aligned}
\mathcal{L}_{1} V(\varphi, i, t) \leq & q|\varphi(0)|^{q-2}\left[\varphi(0)^{T} f(\varphi, i, t)+\frac{q-1}{2}|g(\varphi, i, t)|^{2}\right] \\
\leq & \sum_{k=1}^{2} q \alpha_{k}|\varphi(0)|^{q-2} \int_{-\infty}^{0}|\varphi(\theta)|^{p} d \mu_{k}(\theta)-q \alpha_{3}|\varphi(0)|^{p+q-2} \\
& +\sum_{k=1}^{2} q \beta_{k}|\varphi(0)|^{q-2} \int_{-\infty}^{0}|\varphi(\theta)|^{2} d \mu_{k}(\theta)+q \beta_{3}|\varphi(0)|^{q}
\end{aligned}
$$

Using the Young inequality, we get

$$
\begin{aligned}
q \alpha_{k}|\varphi(0)|^{q-2} & \int_{-\infty}^{0}|\varphi(\theta)|^{p} d \mu_{k}(\theta)=\int_{-\infty}^{0} q \alpha_{k}|\varphi(0)|^{q-2}|\varphi(\theta)|^{p} d \mu_{k}(\theta) \\
\leq & \frac{q \alpha_{k}(q-2)}{p+q-2}|\varphi(0)|^{p+q-2}+\frac{p q \alpha_{k}}{p+q-2} \int_{-\infty}^{0}|\varphi(\theta)|^{p+q-2} d \mu_{k}(\theta), \\
q \beta_{k}|\varphi(0)|^{q-2} & \int_{-\infty}^{0}|\varphi(\theta)|^{2} d \mu_{k}(\theta)=\int_{-\infty}^{0} q \beta_{k}|\varphi(0)|^{q-2}|\varphi(\theta)|^{2} d \mu_{k}(\theta) \\
\leq & (q-2) \beta_{k}|\varphi(0)|^{q}+2 \beta_{k} \int_{-\infty}^{0}|\varphi(\theta)|^{q} d \mu_{k}(\theta) .
\end{aligned}
$$


Substituting these into (2.11) gives

$$
\begin{aligned}
\mathcal{L}_{1} V(\varphi, i, t) \leq & \sum_{k=1}^{2} L_{k}\left(\int_{-\infty}^{0}|\varphi(\theta)|^{p+q-2} d \mu_{k}(\theta)-|\varphi(0)|^{p+q-2}\right) \\
& +2 \sum_{k=1}^{2} \beta_{k}\left(\int_{-\infty}^{0}|\varphi(\theta)|^{q} d \mu_{k}(\theta)-|\varphi(0)|^{q}\right)+C_{1},
\end{aligned}
$$

where

$$
C_{1}=\max _{s \geq 0}\left[-q\left(\alpha_{3}-\alpha_{1}-\alpha_{2}\right) s^{p+q-2}+q\left(\beta_{1}+\beta_{2}+\beta_{3}\right) s^{q}\right], L_{k}=\frac{p q \alpha_{k}}{p+q-2}, k=1,2 .
$$

Step 2. Since the coefficients of the hybrid ISFDE (2.4) are locally Lipschitz continuous, for any given initial data (2.9), using the standard truncation method, there exists a unique maximal local strong solution of Equation (2.4) on $t \in\left(-\infty, \sigma_{e}\right)$, where $\sigma_{e}$ is the explosion time (see, e.g., [36, Theorem 3.2.2, p.95], and [6, Theorem 3.3] ). Let $j_{0}>0$ be a sufficiently large positive number such that $\|\xi\|<j_{0}$. For each integer $j \geq j_{0}$, define the stopping time

$$
\sigma_{j}=\inf \left\{t \in\left[0, \sigma_{e}\right):|x(t)| \geq j\right\},
$$

where throughout this paper we set $\inf \emptyset=\infty$ (as usual $\emptyset$ denotes the empty set). Clearly, $\sigma_{j}$ is increasing as $j \rightarrow \infty$. Set $\sigma_{\infty}=\lim _{j \rightarrow \infty} \sigma_{j}$, whence $\sigma_{\infty} \leq \sigma_{e}$ a.s. If we can show that $\sigma_{\infty}=\infty$ a.s., then $\sigma_{e}=\infty$ a.s., which implies the desired result.

By the Itô formula and (2.11), we obtain

$$
\begin{aligned}
\mathbb{E}\left|x\left(t \wedge \sigma_{j}\right)\right|^{q}= & |x(0)|^{q}+\mathbb{E} \int_{0}^{t \wedge \sigma_{j}} \mathcal{L}_{1} V\left(x_{s}, r(s), s\right) d s \\
\leq & \leq|x(0)|^{q}+\sum_{k=1}^{2} L_{k} \mathbb{E} \int_{0}^{t \wedge \sigma_{j}}\left(\int_{-\infty}^{0}|x(s+\theta)|^{p+q-2} d \mu_{k}(\theta)-|x(s)|^{p+q-2}\right) d s \\
& +2 \sum_{k=1}^{2} \beta_{k} \mathbb{E} \int_{0}^{t \wedge \sigma_{j}}\left(\int_{-\infty}^{0}|x(s+\theta)|^{q} d \mu_{k}(\theta)-|x(s)|^{q}\right) d s+C_{1} t .
\end{aligned}
$$

But

$$
\begin{aligned}
\mathbb{E} \int_{0}^{t \wedge \sigma_{j}} & \left(\int_{-\infty}^{0}|x(s+\theta)|^{p+q-2} d \mu_{k}(\theta)-|x(s)|^{p+q-2}\right) d s \\
= & \mathbb{E} \int_{-\infty}^{0} \int_{0}^{t \wedge \sigma_{j}}|x(s+\theta)|^{p+q-2} d s d \mu_{k}(\theta)-\mathbb{E} \int_{0}^{t \wedge \sigma_{j}}|x(s)|^{p+q-2} d s \\
\quad \leq & \mathbb{E} \int_{-\infty}^{0} \int_{-\infty}^{t \wedge \sigma_{j}}|x(s)|^{p+q-2} d s d \mu_{k}(\theta)-\mathbb{E} \int_{0}^{t \wedge \sigma_{j}}|x(s)|^{p+q-2} d s \\
= & \int_{-\infty}^{0}|\xi(s)|^{p+q-2} d s,
\end{aligned}
$$

and

$$
\begin{aligned}
\mathbb{E} \int_{0}^{t \wedge \sigma_{j}} & \left(\int_{-\infty}^{0}|x(s+\theta)|^{q} d \mu_{k}(\theta)-|x(s)|^{q}\right) d s \\
\quad= & \mathbb{E} \int_{-\infty}^{0} \int_{0}^{t \wedge \sigma_{j}}|x(s+\theta)|^{q} d s d \mu_{k}(\theta)-\mathbb{E} \int_{0}^{t \wedge \sigma_{j}}|x(s)|^{q} d s \\
\quad \leq & \mathbb{E} \int_{-\infty}^{0} \int_{-\infty}^{t \wedge \sigma_{j}}|x(s)|^{q} d s d \mu_{k}(\theta)-\mathbb{E} \int_{0}^{t \wedge \sigma_{j}}|x(s)|^{q} d s \\
\quad= & \int_{-\infty}^{0}|\xi(s)|^{q} d s .
\end{aligned}
$$


Recalling (2.9), by $p+q-2 \geq q \geq 2$, Lemma 2.1 gives that

$$
\int_{-\infty}^{0}|\xi(s)|^{p+q-2} d s \leq\|\xi\|^{p-2} \int_{-\infty}^{0}|\xi(s)|^{q} d s \leq\|\xi\|^{p+q-4} \int_{-\infty}^{0}|\xi(s)|^{2} d s<\infty .
$$

It therefore follow that

$$
\begin{aligned}
\mathbb{E}\left|x\left(t \wedge \sigma_{j}\right)\right|^{q} & \leq|x(0)|^{q}+C_{1} t+\left(L_{1}+L_{2}\right) \int_{-\infty}^{0}|\xi(s)|^{p+q-2} d s+2\left(\beta_{1}+\beta_{2}\right) \int_{-\infty}^{0}|\xi(s)|^{q} d s \\
& =: C(t) .
\end{aligned}
$$

On the other hand, using the definition of stopping time $\sigma_{j}$, we get

$$
C(t) \geq \mathbb{E}\left|x\left(t \wedge \sigma_{j}\right)\right|^{q}=\mathbb{E}\left\{|x(t)|^{q} I_{\sigma_{j}>t}\right\}+\mathbb{E}\left\{j^{q} I_{\sigma_{j} \leq t}\right\} \geq j^{q} P\left(\sigma_{j} \leq t\right) .
$$

Then, we have

$$
P\left(\sigma_{j} \leq t\right) \leq \frac{C(t)}{j^{q}}
$$

Letting $j \rightarrow \infty$, we obtain that $P\left(\sigma_{\infty} \leq t\right)=0$, namely

$$
P\left(\sigma_{\infty}>t\right)=1 .
$$

But $t \geq 0$ is arbitrary, we must have $P\left(\sigma_{\infty}=\infty\right)=1$ as required. The proof is therefore complete.

Obviously, there exists a class of hybrid ISFDEs which satisfy the conditions of Theorem (2.5) but are not stable. In this article, we will focus on how to design the feedback controller to make the unstable ISFDE (2.4) asymptotically bounded and exponentially stable.

Definition 2.6. (i) The equation is said to be asymptotically bounded in qth moment if the solution of the equation satisfies

where $C$ is a positive constant.

$$
\limsup _{t \rightarrow \infty} \mathbb{E}|x(t)|^{q}<C
$$

(ii) The equation is said to be exponentially stable in $L^{p}$ if the solution of the equation satisfies

$$
\limsup _{t \rightarrow \infty} \frac{1}{t} \log \left(\mathbb{E}|x(t)|^{p}\right)<0
$$

(iii) The equation is said to be almost surely exponentially stable if the solution of the equation satisfies

$$
\limsup _{t \rightarrow \infty} \frac{1}{t} \log (|x(t)|)<0 \text { a.s. }
$$

\section{Main results}

When the ISFDE (2.4) is unstable, we need to design a delay feedback controller $u(x(t-\tau), r(t), t)$ to make the original system stable. That is, we will discuss the controlled ISFDE

$$
d x(t)=\left[f\left(x_{t}, r(t), t\right)+u(x(t-\tau), r(t), t)\right] d t+g\left(x_{t}, r(t), t\right) d w(t), \quad t \geq 0,
$$

stability, where the control function $u: R^{n} \times \mathcal{S} \times R_{+} \rightarrow R^{n}$ is a Borel measurable. We always hope that the controller $u$ we designed is more simple and effective, so we give the following condition.

Assumption 3.1. Assume that there is a positive number $\kappa$ such that

$$
|u(x, i, t)-u(y, i, t)| \leq \kappa|x-y|
$$

for all $x, y \in R^{n}, i \in \mathcal{S}$ and $t \geq 0$. Moreover, assume that $u(0, i, t) \equiv 0$ for all $(i, t) \in \mathcal{S} \times R_{+}$.

Obviously this assumption implies

$$
|u(x, i, t)| \leq \kappa|x|, \quad \forall(x, i, t) \in R^{n} \times \mathcal{S} \times R_{+} .
$$




\subsection{Existence, uniqueness and boundedness}

Theorem 3.2. Let Assumptions 2.3, 2.4 and 3.1 hold. For any given initial data (2.9), the new controlled system (3.1) has a unique global solution $x(t)$ on $t \in R$.

Proof. We define a new functional $\mathcal{L}_{2} V: B C\left((-\infty, 0] ; R^{n}\right) \times \mathcal{S} \times R_{+} \rightarrow R$ by

$$
\begin{aligned}
\mathcal{L}_{2} V(\varphi, i, t)= & q|\varphi(0)|^{q-2} \varphi(0)^{T}[f(\varphi, i, t)+u(\varphi(-\tau), i, t)]+\frac{q}{2}|\varphi(0)|^{q-2}|g(\varphi, i, t)|^{2} \\
& +\frac{q(q-2)}{2}|\varphi(0)|^{q-4}\left|\varphi(0)^{T} g(\varphi, i, t)\right|^{2} .
\end{aligned}
$$

Using Assumptions 2.3 and 2.4, we further get

$$
\begin{aligned}
\mathcal{L}_{2} V(\varphi, i, t) \leq & q|\varphi(0)|^{q-2}\left[\varphi(0)^{T} f(\varphi, i, t)+\frac{q-1}{2}|g(\varphi, i, t)|^{2}+\varphi(0)^{T} u(\varphi(-\tau), i, t)\right] \\
\leq & \sum_{k=1}^{2} q \alpha_{k}|\varphi(0)|^{q-2} \int_{-\infty}^{0}|\varphi(\theta)|^{p} d \mu_{k}(\theta)-q \alpha_{3}|\varphi(0)|^{p+q-2}+\sum_{k=1}^{2} q \beta_{k}|\varphi(0)|^{q-2} \\
& \times \int_{-\infty}^{0}|\varphi(\theta)|^{2} d \mu_{k}(\theta)+q \beta_{3}|\varphi(0)|^{q}+q \kappa|\varphi(0)|^{q-1}|\varphi(-\tau)| .
\end{aligned}
$$

Let $v$ be the Dirac measure at $-\tau$. Applying similar technique with (2.12), then using

$$
|\varphi(-\tau)|^{q}=\left|\int_{-\infty}^{0} \varphi(\theta) d v(\theta)\right|^{q} \leq \int_{-\infty}^{0}|\varphi(\theta)|^{q} d v(\theta)
$$

we get

$$
\begin{aligned}
\mathcal{L}_{2} V(\varphi, i, t) \leq & \sum_{k=1}^{2} L_{k} \int_{-\infty}^{0}\left(|\varphi(\theta)|^{p+q-2}-|\varphi(0)|^{p+q-2}\right) d \mu_{k}(\theta)+2 \sum_{k=1}^{2} \beta_{k} \int_{-\infty}^{0}\left(|\varphi(\theta)|^{q}-|\varphi(0)|^{q}\right) d \mu_{k}(\theta) \\
& +\kappa \int_{-\infty}^{0}\left(|\varphi(\theta)|^{q}-|\varphi(0)|^{q}\right) d \nu(\theta)+C_{2}
\end{aligned}
$$

where

$$
C_{2}=\max _{s \geq 0}\left[-q\left(\alpha_{3}-\alpha_{1}-\alpha_{2}\right) s^{p+q-2}+q\left(\beta_{1}+\beta_{2}+\beta_{3}+\kappa\right) s^{q}\right]
$$

Finally, by the same proof method as Theorem 2.5 , we can get this theorem must be hold.

Different from [34], under the assumption of Theorem 3.2, we may only guarantee the existence and uniqueness of the solution of the controlled system (3.1), but not the moment asymptotic boundedness of the solution. However, as long as we want to slightly enhance the condition, we can get the moment asymptotic boundedness.

Theorem 3.3. Let the conditions of Theorem 3.2 hold. Further assume that $\mu_{1}, \mu_{2} \in \mathcal{P}_{\bar{\varepsilon}}$. Then for any given initial data (2.9), the controlled system (3.1) is asymptotically bounded in qth moment. That is, the solution $x(t)$ has the property that

$$
\limsup _{t \rightarrow \infty} \mathbb{E}|x(t)|^{q}<\frac{C_{3}}{\varepsilon},
$$

where

$$
C_{3}=\max _{s \geq 0}\left[-q\left(\alpha_{3}-\alpha_{1} \mu_{1}^{(\varepsilon)}-\alpha_{2} \mu_{2}^{(\varepsilon)}\right) s^{p+q-2}+q\left(\beta_{1} \mu_{1}^{(\varepsilon)}+\beta_{2} \mu_{2}^{(\varepsilon)}+\beta_{3}+\kappa e^{\varepsilon \tau}+\varepsilon\right) s^{q}\right]
$$

and $\varepsilon>0$ is sufficiently small constant such that

$$
\mu_{1}^{(\varepsilon)}=\int_{-\infty}^{0} e^{-\varepsilon \theta} d \mu_{1}(\theta), \mu_{2}^{(\varepsilon)}=\int_{-\infty}^{0} e^{-\varepsilon \theta} d \mu_{2}(\theta) \text { and } \alpha_{1} \mu_{1}^{(\varepsilon)}+\alpha_{2} \mu_{2}^{(\varepsilon)}<\alpha_{3} .
$$


Proof. Firstly, lets show that existence $\varepsilon$ satisfies condition (3.7). The existence of $\mu_{1}^{(\varepsilon)}$ and $\mu_{2}^{(\varepsilon)}$ can be referred to (2.1). From Lemma 2.2 that when $\varepsilon$ monotonically decreases to 0 , there are $\mu_{1}^{(\varepsilon)}$ and $\mu_{2}^{(\varepsilon)}$ also monotonically decrease to 1 . Then, combining with the continuity of $\mu_{1}^{(\varepsilon)}$ and $\mu_{2}^{(\varepsilon)}$ and condition (2.7), there exists $\varepsilon_{2}>0$, which makes any $\varepsilon \in\left(0, \bar{\varepsilon} \wedge \varepsilon_{2}\right)$ condition (3.7) holds.

Recalling (2.7) and (3.7), we can rewrite (3.5) as

$$
\begin{aligned}
\mathcal{L}_{2} V(\varphi, i, t) \leq & \sum_{k=1}^{2} L_{k} \int_{-\infty}^{0}\left(|\varphi(\theta)|^{p+q-2}-\mu_{k}^{(\varepsilon)}|\varphi(0)|^{p+q-2}\right) d \mu_{k}(\theta) \\
& +2 \sum_{k=1}^{2} \beta_{k} \int_{-\infty}^{0}\left(|\varphi(\theta)|^{q}-\mu_{k}^{(\varepsilon)}|\varphi(0)|^{q}\right) d \mu_{k}(\theta) \\
& +\kappa \int_{-\infty}^{0}\left(|\varphi(\theta)|^{q}-e^{\varepsilon \tau}|\varphi(0)|^{q}\right) d v(\theta)-\varepsilon|\varphi(0)|^{q}+C_{3}
\end{aligned}
$$

Applying the Itô formula on function $e^{\varepsilon t}|x|^{q}$, we have

$$
e^{\varepsilon t} \mathbb{E}|x(t)|^{q}=\mathbb{E}|x(0)|^{q}+\mathbb{E} \int_{0}^{t} e^{\varepsilon s}\left[\varepsilon|x(s)|^{q}+\mathcal{L}_{2} V\left(x_{s}, r(s), s\right)\right] d s .
$$

In view of condition (3.8),

$$
\begin{aligned}
e^{\varepsilon t} \mathbb{E}|x(t)|^{q} \leq & |x(0)|^{q}+\sum_{k=1}^{2} L_{k} \mathbb{E} \int_{0}^{t} e^{\varepsilon s}\left(\int_{-\infty}^{0}|x(s+\theta)|^{p+q-2} d \mu_{k}(\theta)-\mu_{k}^{(\varepsilon)}|x(s)|^{p+q-2}\right) d s \\
& +2 \sum_{k=1}^{2} \beta_{k} \mathbb{E} \int_{0}^{t} e^{\varepsilon s}\left(\int_{-\infty}^{0}|x(s+\theta)|^{q} d \mu_{k}(\theta)-\mu_{k}^{(\varepsilon)}|x(s)|^{q}\right) d s \\
& +\kappa \mathbb{E} \int_{0}^{t} e^{\varepsilon s}\left(\int_{-\infty}^{0}|x(s+\theta)|^{q} d v(\theta)-e^{\varepsilon \tau}|x(s)|^{q}\right) d s+\frac{C_{3} e^{\varepsilon t}}{\varepsilon} .
\end{aligned}
$$

By the Fubini theorem and a substitution technique, it is easy to show that

$$
\begin{aligned}
& \mathbb{E} \int_{0}^{t} e^{\varepsilon s}\left(\int_{-\infty}^{0}|x(s+\theta)|^{p+q-2} d \mu_{k}(\theta)-\mu_{k}^{(\varepsilon)}|x(s)|^{p+q-2}\right) d s \\
& \quad \leq \mathbb{E} \int_{-\infty}^{0} \int_{0}^{t} e^{\varepsilon s}|x(s+\theta)|^{p+q-2} d s d \mu_{k}(\theta)-\mu_{k}^{(\varepsilon)} \mathbb{E} \int_{0}^{t} e^{\varepsilon s}|x(s)|^{p+q-2} d s \\
& \quad \leq \mathbb{E} \int_{-\infty}^{0} e^{-\varepsilon \theta} d \mu_{k}(\theta) \int_{-\infty}^{t} e^{\varepsilon s}|x(s)|^{p+q-2} d s-\mu_{k}^{(\varepsilon)} \mathbb{E} \int_{0}^{t} e^{\varepsilon s}|x(s)|^{p+q-2} d s \\
& \quad=\mu_{k}^{(\varepsilon)} \int_{-\infty}^{0} e^{\varepsilon s}|\xi(s)|^{p+q-2} d s \leq \mu_{k}^{(\varepsilon)} \int_{-\infty}^{0}|\xi(s)|^{p+q-2} d s .
\end{aligned}
$$

Similarly, we then derive

$$
\mathbb{E} \int_{0}^{t} e^{\varepsilon s}\left(\int_{-\infty}^{0}|x(s+\theta)|^{q} d \mu_{k}(\theta)-\mu_{k}^{(\varepsilon)}|x(s)|^{q}\right) d s \leq \mu_{k}^{(\varepsilon)} \int_{-\infty}^{0}|\xi(s)|^{q} d s
$$

and

$$
\mathbb{E} \int_{0}^{t} e^{\varepsilon s}\left(\int_{-\infty}^{0}|x(s+\theta)|^{q} d v(\theta)-e^{\varepsilon \tau}|x(s)|^{q}\right) d s \leq e^{\varepsilon \tau} \mathbb{E} \int_{-\infty}^{0}|\xi(s)|^{q} d s
$$


Substituting these into (3.9) give

$$
\begin{aligned}
e^{\varepsilon t} \mathbb{E}|x(t)|^{q} \leq & |x(0)|^{q}+\frac{C_{3} e^{\varepsilon t}}{\varepsilon}+\sum_{k=1}^{2} L_{k} \mu_{k}^{(\varepsilon)} \int_{-\infty}^{0}|\xi(s)|^{p+q-2} d s \\
& +2 \sum_{k=1}^{2} \beta_{k} \mu_{k}^{(\varepsilon)} \int_{-\infty}^{0}|\xi(s)|^{q} d s+e^{\varepsilon \tau} \int_{-\infty}^{0}|\xi(s)|^{q} d s=: \frac{C_{3} e^{\varepsilon t}}{\varepsilon}+C_{4} .
\end{aligned}
$$

Dividing both sides by $e^{\varepsilon t}$, and letting $t \rightarrow \infty$, we obtain the assertion (3.6).

This theorem implies a number of good properties of the solution. For example, $\sup _{t \geq 0} \mathbb{E}|x(t)|^{\bar{q}}<\infty$ for any $\bar{q} \in(0, q]$ while both $f\left(x_{t}, r(t), t\right)$ and $g\left(x_{t}, r(t), t\right)$ are bounded in $L^{2}$ on $t \geq 0$.

\subsection{Exponential stabilization}

However, only Assumption 3.1 can not guarantee the stability of the controlled system (3.1), we need to give more criteria related to the control function $u$. In this paper, we will use M-matrix to construct Lyapunov functional to obtain exponential stability of the ISFDE (3.1). Regarding the theory on M-matrix we refer the reader to [37]. Now we give the first criterion, which is related to M-matrix.

Assumption 3.4. For each $i \in S$, there exist nonnegative numbers $\alpha_{i k}, \hat{\alpha}_{i k}, \beta_{i k}, \hat{\beta}_{i k}(k=1,2)$, positive numbers $\alpha_{i 3}, \hat{\alpha}_{i 3}$ and real numbers $\beta_{i 3}, \hat{\beta}_{i 3}$ for both

$$
\begin{aligned}
\varphi(0)^{T}[f(\varphi, i, t)+ & u(\varphi(0), i, t)]+\frac{1}{2}|g(\varphi, i, t)|^{2} \leq \sum_{k=1}^{2} \alpha_{i k} \int_{-\infty}^{0}|\varphi(\theta)|^{p} d \mu_{k}(\theta) \\
& -\alpha_{i 3}|\varphi(0)|^{p}+\sum_{k=1}^{2} \beta_{i k} \int_{-\infty}^{0}|\varphi(\theta)|^{2} d \mu_{k}(\theta)+\beta_{i 3}|\varphi(0)|^{2}
\end{aligned}
$$

and

$$
\begin{aligned}
\varphi(0)^{T}[f(\varphi, i, t)+ & u(\varphi(0), i, t)]+\frac{q_{1}}{2}|g(\varphi, i, t)|^{2} \leq \sum_{k=1}^{2} \hat{\alpha}_{i k} \int_{-\infty}^{0}|\varphi(\theta)|^{p} d \mu_{k}(\theta) \\
& -\hat{\alpha}_{i 3}|\varphi(0)|^{p}+\sum_{k=1}^{2} \hat{\beta}_{i k} \int_{-\infty}^{0}|\varphi(\theta)|^{2} d \mu_{k}(\theta)+\hat{\beta}_{i 3}|\varphi(0)|^{2}
\end{aligned}
$$

to hold for all $(\varphi, i, t) \in B C\left((-\infty, 0] ; R^{n}\right) \times \mathcal{S} \times R_{+}$(where $q_{1}$ has been specified in Assumption 2.3). In addition, both

$$
\begin{aligned}
& \mathcal{A}_{1}:=-2 \operatorname{diag}\left(\beta_{13}, \cdots, \beta_{N 3}\right)-\Gamma, \\
& \mathcal{A}_{2}:=-\left(q_{1}+1\right) \operatorname{diag}\left(\hat{\beta}_{12}, \cdots, \hat{\beta}_{N 2}\right)-\Gamma
\end{aligned}
$$

are nonsingular M-matrices.

We will explain that there are many control functions that can satisfy Assumption 3.1 and make Assumption 3.4 hold at the same time. For example, let's take a linear controller $u(x, i, t)=A x$, where $A$ is a symmetric $n \times n$ real-valued negative-definite matrix such that $\lambda_{\max }(A) \leq-2 \beta_{3}$ (obviously satisfies Assumption 3.1). Then

$$
x^{T} u(x, i, t) \leq-2 \beta_{3}|x|^{2}, \quad \forall(x, i, t) \in R^{n} \times \mathcal{S} \times R_{+} .
$$

Combining this and (2.8), we deduce that

$$
\begin{aligned}
& \varphi(0)^{T}[f(\varphi, i, t)+u(\varphi(0), i, t)]+\frac{1}{2}|g(\varphi, i, t)|^{2} \\
& \leq \sum_{k=1}^{2} \alpha_{k} \int_{-\infty}^{0}|\varphi(\theta)|^{p} d \mu_{k}(\theta)-\alpha_{3}|\varphi(0)|^{p}+\sum_{k=1}^{2} \beta_{k} \int_{-\infty}^{0}|\varphi(\theta)|^{2} d \mu_{k}(\theta)-\beta_{3}|\varphi(0)|^{2}
\end{aligned}
$$


as well as

$$
\begin{aligned}
& \varphi(0)^{T} {[f(\varphi, i, t)+u(\varphi(0), i, t)]+\frac{q_{1}}{2}|g(\varphi, i, t)|^{2} } \\
& \leq \sum_{k=1}^{2} \alpha_{k} \int_{-\infty}^{0}|\varphi(\theta)|^{p} d \mu_{k}(\theta)-\alpha_{3}|\varphi(0)|^{p}+\sum_{k=1}^{2} \beta_{k} \int_{-\infty}^{0}|\varphi(\theta)|^{2} d \mu_{k}(\theta)-\beta_{3}|\varphi(0)|^{2}
\end{aligned}
$$

while

$$
\mathcal{A}_{1}=2 \operatorname{diag}\left(\beta_{3}, \cdots, \beta_{3}\right)-\Gamma \text { and } \mathcal{A}_{2}=\left(q_{1}+1\right) \operatorname{diag}\left(\beta_{3}, \cdots, \beta_{3}\right)-\Gamma
$$

which are nonsingular M-matrices. That is, the control function $u(x, i, t)=A x$ meets Assumption 3.4.

We set

$$
\begin{aligned}
& \left(c_{1}, \cdots, c_{N}\right)^{T}:=\mathcal{A}_{1}^{-1}(1, \cdots, 1)^{T}, \\
& \left(\hat{c}_{1}, \cdots, \hat{c}_{N}\right)^{T}:=\mathcal{A}_{2}^{-1}(1, \cdots, 1)^{T},
\end{aligned}
$$

where $\mathcal{A}_{1}$ and $\mathcal{A}_{2}$ have specified in Assumption 3.4. Obviously, both $c_{i}$ and $\hat{c}_{i}$ are positive numbers. Define a function $U: R^{n} \times \mathcal{S} \rightarrow R_{+}$by

$$
U(x, i)=c_{i}|x|^{2}+\hat{c}_{i}|x|^{q_{1}+1}, \quad(x, i) \in R^{n} \times \mathcal{S}
$$

while define a functional $\mathcal{L} U: B C\left((-\infty, 0] ; R^{n}\right) \times \mathcal{S} \times R_{+} \rightarrow R$ by

$$
\begin{aligned}
& \mathcal{L} U(\varphi, i, t)=2 c_{i}\left[\varphi(0)^{T}[f(\varphi, i, t)+u(\varphi(0), i, t)]+\frac{1}{2}|g(\varphi, i, t)|^{2}\right]+\left(q_{1}+1\right) \hat{c}_{i}|\varphi(0)|^{q_{1}-1} \\
& \times\left[\varphi(0)^{T}[f(\varphi, i, t)+u(\varphi(0), i, t)]+\frac{q_{1}}{2}|g(\varphi, i, t)|^{2}\right]+\sum_{j=1}^{N} \gamma_{i j}\left(c_{j}|\varphi(0)|^{2}+\hat{c}_{j}|\varphi(0)|^{q_{1}+1}\right) .
\end{aligned}
$$

Next, we will use $\mathcal{L} U(\varphi, i, t)$ to give the second stability criterion.

Assumption 3.5. Assume that there exists a function $\Phi(x) \in C\left(R^{n} ; R_{+}\right)$, as well as positive numbers $\gamma_{j}(j=1,2, \ldots, 9)$, such that

$$
\gamma_{4}+\gamma_{5}<1, \quad \gamma_{6}+\gamma_{7}<1, \quad \gamma_{8}|x|^{p+q_{1}-1} \leq \Phi(x) \leq \gamma_{9}\left(|x|^{2}+|x|^{p+q_{1}-1}\right)
$$

and

$$
\begin{aligned}
\mathcal{L} U(\varphi, i, t) & +\gamma_{1}\left(2 c_{i}|\varphi(0)|+\left(q_{1}+1\right) \hat{c}_{i}|\varphi(0)|^{q_{1}}\right)^{2}+\gamma_{2}|f(\varphi, i, t)|^{2}+\gamma_{3}|g(\varphi, i, t)|^{2} \\
\leq & -\rho\left(|\varphi(0)|^{2}-\gamma_{4} \int_{-\infty}^{0}|\varphi(\theta)|^{2} d \mu_{1}(\theta)-\gamma_{5} \int_{-\infty}^{0}|\varphi(\theta)|^{2} d \mu_{2}(\theta)\right) \\
& -\Phi(\varphi(0))+\gamma_{6} \int_{-\infty}^{0} \Phi(\varphi(\theta)) d \mu_{1}(\theta)+\gamma_{7} \int_{-\infty}^{0} \Phi(\varphi(\theta)) d \mu_{2}(\theta)
\end{aligned}
$$

for all $(\varphi, i, t) \in B C\left((-\infty, 0] ; R^{n}\right) \times \mathcal{S} \times R_{+}$.

The Lyapunov functional used in this paper will be of the form

$$
\begin{aligned}
\bar{V}\left(x_{t}, r_{t}, t\right)= & U(x(t), r(t))+\vartheta \int_{-\tau}^{0} \int_{t+s}^{t}\left[\tau\left|f\left(x_{v}, r(v), v\right)+u(x(v-\tau), r(v), v)\right|^{2}\right. \\
& \left.+\left|g\left(x_{v}, r(v), v\right)\right|^{2}\right] d v d s
\end{aligned}
$$


for $t \geq 0$, where $U$ has been defined by (3.16), $r_{t}:=\{r(t+s):-\tau \leq s \leq 0\}$ and $\vartheta$ is a positive constant to be determined later. For $r_{t}$ to be well defined for $0 \leq t<\tau$, we set $r(s)=r(0)=i_{0}$ for $s \in[-\tau, 0)$. By the generalized Itô formula and simple differential calculations, we get

$$
d \bar{V}\left(x_{t}, r_{t}, t\right)=\left(L U\left(x_{t}, r(t), t\right)+\vartheta H\left(x_{t}, r_{t}, t\right)\right) d t+d M(t)
$$

for $t \geq 0$, where $M(t)$ is a continuous local martingale with $M(0)=0$ (see, e.g., [37, Theorem 1.45 on p.48]), $L U: B C\left((-\infty, 0] ; R^{n}\right) \times \mathcal{S} \times R_{+} \rightarrow R$ and $H\left(x_{t}, r_{t}, t\right)$ are defined as

$$
\begin{aligned}
& L U\left(x_{t}, r(t), t\right)=2 c_{r(t)}\left[x(t)^{T}\left[f\left(x_{t}, r(t), t\right)+u(x(t-\tau), r(t), t]+\frac{1}{2}\left|g\left(x_{t}, r(t), t\right)\right|^{2}\right]\right. \\
& +\left(q_{1}+1\right) \hat{c}_{r(t)}|x(t)|^{q_{1}-1}\left[x(t)^{T}\left[f\left(x_{t}, r(t), t\right)+u(x(t-\tau), r(t), t)\right]+\frac{1}{2}\left|g\left(x_{t}, r(t), t\right)\right|^{2}\right] \\
& +\frac{\left(q_{1}^{2}-1\right)}{2} \hat{c}_{r(t)}|x(t)|^{q_{1}-3}\left|x(t)^{T} g\left(x_{t}, r(t), t\right)\right|^{2}+\sum_{j=1}^{N} \gamma_{r(t) j}\left(c_{j}|x(t)|^{2}+\hat{c}_{j}|x(t)|^{q_{1}+1}\right),
\end{aligned}
$$

and

$$
\begin{aligned}
& H\left(x_{t}, r_{t}, t\right)=\tau\left[\tau\left|f\left(x_{t}, r(t), t\right)+u(x(t-\tau), r(t), t)\right|^{2}+\left|g\left(x_{t}, r(t), t\right)\right|^{2}\right] \\
&-\int_{t-\tau}^{t}\left[\tau\left|f\left(x_{v}, r(v), v\right)+u(x(v-\tau), r(v), v)\right|^{2}+\left|g\left(x_{v}, r(v), v\right)\right|^{2}\right] d v
\end{aligned}
$$

Obviously, we deduce that

$$
\begin{aligned}
L U\left(x_{t}, i, t\right) & \leq 2 c_{i}\left[x(t)^{T}\left[f\left(x_{t}, i, t\right)+u(x(t-\tau), i, t]+\frac{1}{2}\left|g\left(x_{t}, i, t\right)\right|^{2}\right]+\left(q_{1}+1\right) \hat{c}_{i}|x(t)|^{q_{1}-1}\right. \\
\times & {\left[x(t)^{T}\left[f\left(x_{t}, i, t\right)+u(x(t-\tau), i, t)\right]+\frac{q_{1}}{2}\left|g\left(x_{t}, i, t\right)\right|^{2}\right]+\sum_{j=1}^{N} \gamma_{i j}\left(c_{j}|x(t)|^{2}+\hat{c}_{j}|x(t)|^{q_{1}+1}\right) } \\
= & \mathcal{L} U\left(x_{t}, i, t\right)+\left[2 c_{i}+\left(q_{1}+1\right) \hat{c}_{i}|x(t)|^{q_{1}-1}\right] x(t)^{T}[u(x(t-\tau), i, t)-u(x(t), i, t)] .
\end{aligned}
$$

We can now state our first stabilization result.

Theorem 3.6. Let Assumptions 2.3, 2.4, 3.1, 3.4, 3.5 hold. Further assume that $\mu_{1}, \mu_{2} \in \mathcal{P}_{\hat{\varepsilon}}$. Assume also $\tau$ is sufficiently small for

$$
\tau<\frac{\sqrt{3 \rho \gamma_{1}\left(1-\gamma_{4}-\gamma_{5}\right)}}{2 \kappa^{2}} \text { and } \tau \leq \frac{\sqrt{3 \gamma_{1} \gamma_{2}}}{2 \kappa} \wedge \frac{3 \gamma_{1} \gamma_{3}}{2 \kappa^{2}}
$$

Then the solution of the controlled system (3.1) satisfies

$$
\limsup _{t \rightarrow \infty} \frac{1}{t} \log \left(\mathbb{E}|x(t)|^{2}\right)<0
$$

for any initial data (2.9). That is, the controlled system (3.1) is exponentially stable in $L^{2}$.

Proof. Similar to the first paragraph of Theorem 3.3, since (3.18) and (3.24), combined with the continuity of $\mu_{1}^{(\varepsilon)}$ and $\mu_{2}^{(\varepsilon)}$, there is $\varepsilon_{3}>0$, and

$$
\rho \gamma_{4} \mu_{1}^{(\varepsilon)}+\rho \gamma_{5} \mu_{2}^{(\varepsilon)}<\rho-\frac{4 \tau^{2} \kappa^{4}}{3 \gamma_{1}}, \quad \gamma_{6} \mu_{1}^{(\varepsilon)}+\gamma_{7} \mu_{2}^{(\varepsilon)}<1
$$

hold for any $\varepsilon \in\left(0, \hat{\varepsilon} \wedge \varepsilon_{3}\right)$. To make the proof more understandable, we divide it into the following three steps.

Step 1. Set

$$
\mathcal{L} \bar{V}\left(x_{t}, r_{t}, t\right)=L U\left(x_{t}, r(t), t\right)+\vartheta H\left(x_{t}, r_{t}, t\right) .
$$


Let $\vartheta=2 \kappa^{2} / 3 \gamma_{1}$. By condition (3.24), it is easy to show that $\frac{4 \kappa^{2} \tau^{2}}{3 \gamma_{1}} \leq \gamma_{2}, \frac{2 \kappa^{2} \tau}{3 \gamma_{1}} \leq \gamma_{3}$. Using the basic inequality, we get

$$
\begin{aligned}
\vartheta H\left(x_{t}, r_{t}, t\right) \leq & \gamma_{2}\left|f\left(x_{t}, r(t), t\right)\right|^{2}+\gamma_{3}\left|g\left(x_{t}, r(t), t\right)\right|^{2}+\frac{4 \tau^{2} \kappa^{2}}{3 \gamma_{1}}|u(x(t-\tau), r(t), t)|^{2} \\
& -\frac{2 \kappa^{2}}{3 \gamma_{1}} \int_{t-\tau}^{t}\left[\tau\left|f\left(x_{v}, r(v), v\right)+u(x(v-\tau), r(v), v)\right|^{2}+\left|g\left(x_{v}, r(v), v\right)\right|^{2}\right] d v .
\end{aligned}
$$

On the other hand, combining (3.23) and Assumption 3.1, we have

$$
L U\left(x_{t}, r(t), t\right) \leq \mathcal{L} U\left(x_{t}, r(t), t\right)+\gamma_{1}\left[2 c_{r(t)}|x(t)|+\left(q_{1}+1\right) \hat{c}_{r(t)}|x(t)|^{q_{1}}\right]^{2}+\frac{\kappa^{2}}{4 \gamma_{1}}|x(t-\tau)-x(t)|^{2} .
$$

Plugging these into (3.27), then using conditions (3.3) and (3.19), we obtain that

$$
\begin{aligned}
\mathcal{L} \bar{V}\left(x_{t}, r_{t}, t\right) \leq & \mathcal{L} U\left(x_{t}, r(t), t\right)+\gamma_{1}\left[c_{r(t)}|x(t)|+\left(q_{1}+1\right) \hat{c}_{r(t)}|x(t)|^{q_{1}}\right]^{2}+\gamma_{2}\left|f\left(x_{t}, r(t), t\right)\right|^{2} \\
& +\gamma_{3}\left|g\left(x_{t}, r(t), t\right)\right|^{2}+\frac{4 \tau^{2} \kappa^{2}}{3 \gamma_{1}}|u(x(t-\tau), r(t), t)|^{2}+\frac{\kappa^{2}}{4 \gamma_{1}}|x(t-\tau)-x(t)|^{2} \\
& -\frac{2 \kappa^{2}}{3 \gamma_{1}} \int_{t-\tau}^{t}\left[\tau\left|f\left(x_{v}, r(v), v\right)+u(x(v-\tau), r(v), v)\right|^{2}+\left|g\left(x_{v}, r(v), v\right)\right|^{2}\right] d v \\
\leq & -\rho\left(|x(t)|^{2}-\gamma_{4} \int_{-\infty}^{0}|x(t+\theta)|^{2} d \mu_{1}(\theta)-\gamma_{5} \int_{-\infty}^{0}|x(t+\theta)|^{2} d \mu_{2}(\theta)\right) \\
& -\Phi(x(t))+\gamma_{6} \int_{-\infty}^{0} \Phi(x(t+\theta)) d \mu_{1}(\theta)+\gamma_{7} \int_{-\infty}^{0} \Phi(x(t+\theta)) d \mu_{2}(\theta) \\
& +\frac{4 \tau^{2} \kappa^{4}}{3 \gamma_{1}}|x(t-\tau)|^{2}+\frac{\kappa^{2}}{4 \gamma_{1}}|x(t-\tau)-x(t)|^{2} \\
& -\frac{2 \kappa^{2}}{3 \gamma_{1}} \int_{t-\tau}^{t}\left[\tau\left|f\left(x_{v}, r(v), v\right)+u(x(v-\tau), r(v), v)\right|^{2}+\left|g\left(x_{v}, r(v), v\right)\right|^{2}\right] d v .
\end{aligned}
$$

By Assumptions 2.3, 2.4 and 3.1 as well as Theorem 3.3, it is straightforward to see that

$$
\sup _{t \geq 0} \mathbb{E}\left|\mathcal{L} \bar{V}\left(x_{t}, r_{t}, t\right)\right|<\infty .
$$

Step 2. Combining the generalized Itô formula and (3.29), we obtain

$$
e^{\varepsilon t} \mathbb{E} \bar{V}\left(x_{t}, r_{t}, t\right)=\bar{V}\left(x_{0}, r_{0}, 0\right)+\mathbb{E} \int_{0}^{t} e^{\varepsilon s}\left(\varepsilon \bar{V}\left(x_{s}, r_{s}, s\right)+\mathcal{L} \bar{V}\left(x_{s}, r_{s}, s\right)\right) d s
$$

for any $t \geq 0$. Recalling (3.18), we have

$$
|x(s)|^{q_{1}+1} \leq|x(s)|^{2}+|x(s)|^{p+q_{1}-1} \leq|x(s)|^{2}+\gamma_{8}^{-1} \Phi(x(s)) .
$$

Set $\lambda_{1}=\min _{i \in \mathcal{S}} c_{i}, \lambda_{2}=\max _{i \in \mathcal{S}} c_{i}, \lambda_{3}=\max _{i \in \mathcal{S}} \hat{c}_{i}$. We can rewrite (3.30) as

$$
\begin{aligned}
\lambda_{1} e^{\varepsilon t} \mathbb{E}|x(t)|^{2} \leq e^{\varepsilon t} \mathbb{E} \bar{V}\left(x_{t}, r_{t}, t\right) & \leq \bar{V}\left(x_{0}, r_{0}, 0\right)+\mathbb{E} \int_{0}^{t} e^{\varepsilon s}\left(\varepsilon\left(\lambda_{2}+\lambda_{3}\right)|x(s)|^{2}\right. \\
& \left.+\frac{\varepsilon \lambda_{3}}{\gamma_{8}} \Phi(x(s))+\mathcal{L} \bar{V}\left(x_{s}, r_{s}, s\right)\right) d s+J
\end{aligned}
$$

where

$$
J=\varepsilon \vartheta \mathbb{E} \int_{0}^{t} e^{\varepsilon s}\left(\int_{-\tau}^{0} \int_{s+w}^{s}\left[\tau\left|f\left(x_{v}, r(v), v\right)+u(x(v-\tau), r(v), v)\right|^{2}+\left|g\left(x_{v}, r(v), v\right)\right|^{2}\right] d v d w\right) d s
$$


Substituting (3.28) into (3.31), yields

$$
\lambda_{1} e^{\varepsilon t} \mathbb{E}|x(t)|^{2} \leq \bar{V}\left(x_{0}, r_{0}, 0\right)+I_{1}+I_{2}+I_{3}-I_{4}+J,
$$

where

$$
\begin{aligned}
I_{1}= & \mathbb{E} \int_{0}^{t} e^{\varepsilon s}\left[-\left(\rho-\varepsilon \lambda_{2}-\varepsilon \lambda_{3}\right)|x(s)|^{2}+\frac{4 \tau^{2} \kappa^{4}}{3 \gamma_{1}}|x(s-\tau)|^{2}+\rho \gamma_{4} \int_{-\infty}^{0}|x(s+\theta)|^{2} d \mu_{1}(\theta)\right. \\
& \left.+\rho \gamma_{5} \int_{-\infty}^{0}|x(s+\theta)|^{2} d \mu_{2}(\theta)\right] d s \\
I_{2}= & \mathbb{E} \int_{0}^{t} e^{\varepsilon s}\left[-\left(1-\frac{\varepsilon \lambda_{3}}{\gamma_{8}}\right) \Phi(x(s))+\gamma_{6} \int_{-\infty}^{0} \Phi(x(s+\theta)) d \mu_{1}(\theta)+\gamma_{7} \int_{-\infty}^{0} \Phi(x(s+\theta)) d \mu_{2}(\theta)\right] d s, \\
I_{3}= & \frac{\kappa^{2}}{4 \gamma_{1}} \mathbb{E} \int_{0}^{t} e^{\varepsilon s}|x(s-\tau)-x(s)|^{2} d s, \\
I_{4}= & \frac{2 \kappa^{2}}{3 \gamma_{1}} \mathbb{E} \int_{0}^{t} e^{\varepsilon s}\left(\int_{s-\tau}^{s}\left[\tau\left|f\left(x_{v}, r(v), v\right)+u(x(v-\tau), r(v), v)\right|^{2}+\left|g\left(x_{v}, r(v), v\right)\right|^{2}\right] d v\right) d s .
\end{aligned}
$$

Step 3. By the substitution technique, we deduce that

$$
\begin{aligned}
\int_{0}^{t} \int_{-\infty}^{0} e^{\varepsilon s}|x(s+\theta)|^{2} d \mu_{k}(\theta) d s & =\int_{-\infty}^{0} e^{-\varepsilon \theta} \int_{0}^{t} e^{\varepsilon(s+\theta)}|x(s+\theta)|^{2} d s d \mu_{k}(\theta) \\
& \leq \int_{-\infty}^{0} e^{-\varepsilon \theta} d \mu_{k}(\theta) \int_{-\infty}^{t} e^{\varepsilon s}|x(s)|^{2} d s \\
& \leq \mu_{k}^{(\varepsilon)}\left(\int_{-\infty}^{0}|\xi(s)|^{2} d s+\int_{0}^{t} e^{\varepsilon s}|x(s)|^{2} d s\right)
\end{aligned}
$$

and

$$
\int_{0}^{t} e^{\varepsilon s}|x(s-\tau)|^{2} d s=e^{\varepsilon \tau} \int_{-\tau}^{t-\tau} e^{\varepsilon s}|x(s)|^{2} d s \leq e^{\varepsilon \tau}\left(\int_{-\infty}^{0}|\xi(s)|^{2} d s+\int_{0}^{t} e^{\varepsilon s}|x(s)|^{2} d s\right) .
$$

Thus

$$
\begin{aligned}
I_{1} \leq & \left(\rho\left(\gamma_{4} \mu_{1}^{(\varepsilon)}+\gamma_{5} \mu_{2}^{(\varepsilon)}\right)+\frac{4 \tau^{2} \kappa^{4} e^{\varepsilon \tau}}{3 \gamma_{1}}\right) \int_{-\infty}^{0}|\xi(s)|^{2} d s \\
& -\left(\rho-\varepsilon \lambda_{2}-\varepsilon \lambda_{3}-\frac{4 \tau^{2} \kappa^{4} e^{\varepsilon \tau}}{3 \gamma_{1}}-\rho\left(\gamma_{4} \mu_{1}^{(\varepsilon)}+\gamma_{5} \mu_{2}^{(\varepsilon)}\right)\right) \mathbb{E} \int_{0}^{t} e^{\varepsilon s}|x(s)|^{2} d s .
\end{aligned}
$$

Similarly, and then use (3.18) to get

$$
\begin{aligned}
I_{2} \leq & \left(\gamma_{6} \mu_{1}^{(\varepsilon)}+\gamma_{7} \mu_{2}^{(\varepsilon)}\right) \int_{-\infty}^{0} \Phi(\xi(s)) d s-\left(1-\frac{\varepsilon \lambda_{3}}{\gamma_{8}}-\left(\gamma_{6} \mu_{1}^{(\varepsilon)}+\gamma_{7} \mu_{2}^{(\varepsilon)}\right)\right) \mathbb{E} \int_{0}^{t} e^{\varepsilon s} \Phi(x(s)) d s \\
& \leq \gamma_{9}\left(\gamma_{6} \mu_{1}^{(\varepsilon)}+\gamma_{7} \mu_{2}^{(\varepsilon)}\right)\left(\int_{-\infty}^{0}|\xi(s)|^{2} d s+\int_{-\infty}^{0}|\xi(s)|^{p+q_{1}-1} d s\right) \\
& -\left(1-\frac{\varepsilon \lambda_{3}}{\gamma_{8}}-\left(\gamma_{6} \mu_{1}^{(\varepsilon)}+\gamma_{7} \mu_{2}^{(\varepsilon)}\right)\right) \mathbb{E} \int_{0}^{t} e^{\varepsilon s} \Phi(x(s)) d s .
\end{aligned}
$$

On the other hand, by the Fubini theorem,

$$
I_{3}=\frac{\kappa^{2}}{4 \gamma_{1}} \int_{0}^{t} e^{\varepsilon s} \mathbb{E}|x(s)-x(s-\tau)|^{2} d s
$$


For $t \in[0, \tau]$, we have

$$
I_{3} \leq \frac{\kappa^{2}}{4 \gamma_{1}} \int_{0}^{\tau} e^{\varepsilon s} \mathbb{E}|x(s)-x(s-\tau)|^{2} d s \leq \frac{\tau e^{\varepsilon \tau} \kappa^{2}}{\gamma_{1}} \sup _{v \in[-\tau, \tau]} \mathbb{E}|x(v)|^{2}=: C_{5}
$$

For $t>\tau$, using the Hölder inequality and the Itô isometry, we derive that

$$
\begin{aligned}
I_{3} & <C_{5}+\frac{\kappa^{2}}{4 \gamma_{1}} \int_{\tau}^{t} e^{\varepsilon s} \mathbb{E}|x(s)-x(s-\tau)|^{2} d s \\
& \leq C_{5}+\frac{\kappa^{2}}{2 \gamma_{1}} \int_{\tau}^{t} e^{\varepsilon s} \mathbb{E} \int_{s-\tau}^{s}\left(\tau\left|f\left(x_{v}, r(v), v\right)+u(x(v-\tau), r(v), v)\right|^{2}+\left|g\left(x_{v}, r(v), v\right)\right|^{2}\right) d v \\
& \leq C_{5}+3 / 4 I_{4} .
\end{aligned}
$$

This implies

$$
I_{3} \leq C_{5}+3 / 4 I_{4}
$$

It is straightforward to show that

$$
\begin{aligned}
J & \leq \frac{2 \varepsilon \kappa^{2}}{3 \gamma_{1}} \mathbb{E} \int_{0}^{t} e^{\varepsilon s}\left(\tau \int_{s-\tau}^{s}\left[\tau\left|f\left(x_{v}, r(v), v\right)+u(x(v-\tau), r(v), v)\right|^{2}+\left|g\left(x_{v}, r(v), v\right)\right|^{2}\right] d v\right) d s \\
& =\varepsilon \tau I_{4} .
\end{aligned}
$$

Substituting (3.33), (3.34), (3.35) and (3.36) into (3.32), we have

$$
\begin{aligned}
\lambda_{1} e^{\varepsilon t} \mathbb{E}|x(t)|^{2} \leq & C_{6}-\left(\rho-\varepsilon \lambda_{2}-\varepsilon \lambda_{3}-\frac{4 \tau^{2} \kappa^{4} e^{\varepsilon \tau}}{3 \gamma_{1}}-\rho\left(\gamma_{4} \mu_{1}^{(\varepsilon)}+\gamma_{5} \mu_{2}^{(\varepsilon)}\right)\right) \mathbb{E} \int_{0}^{t} e^{\varepsilon s}|x(s)|^{2} d s \\
& -\left(1-\frac{\varepsilon \lambda_{3}}{\gamma_{8}}-\left(\gamma_{6} \mu_{1}^{(\varepsilon)}+\gamma_{7} \mu_{2}^{(\varepsilon)}\right)\right) \mathbb{E} \int_{0}^{t} e^{\varepsilon s} \Phi(x(s)) d s-(1 / 4-\varepsilon \tau) I_{4},
\end{aligned}
$$

where $C_{6}$ is a constant defined by

$$
\begin{aligned}
C_{6}= & \bar{V}\left(x_{0}, r_{0}, 0\right)+\left(\rho\left(\gamma_{4} \mu_{1}^{(\varepsilon)}+\gamma_{5} \mu_{2}^{(\varepsilon)}\right)+\frac{4 \tau^{2} \kappa^{4} e^{\varepsilon \tau}}{3 \gamma_{1}}+\gamma_{9}\left(\gamma_{6} \mu_{1}^{(\varepsilon)}+\gamma_{7} \mu_{2}^{(\varepsilon)}\right)\right) \int_{-\infty}^{0}|\xi(s)|^{2} d s \\
& +\gamma_{9}\left(\gamma_{6} \mu_{1}^{(\varepsilon)}+\gamma_{7} \mu_{2}^{(\varepsilon)}\right) \int_{-\infty}^{0}|\xi(s)|^{p+q_{1}-1} d s+C_{5} .
\end{aligned}
$$

Recalling (3.26), we may make sure $\varepsilon \in\left(0, \hat{\varepsilon} \wedge \varepsilon_{3}\right)$ to be sufficiently small for

$$
\begin{aligned}
& \rho \gamma_{4} \mu_{1}^{(\varepsilon)}+\rho \gamma_{5} \mu_{2}^{(\varepsilon)}+\varepsilon \lambda_{2}+\varepsilon \lambda_{3}+\frac{4 \tau^{2} \kappa^{4} e^{\varepsilon \tau}}{3 \gamma_{1}} \leq \rho, \\
& \gamma_{6} \mu_{1}^{(\varepsilon)}+\gamma_{7} \mu_{2}^{(\varepsilon)}+\frac{\varepsilon \lambda_{3}}{\gamma_{8}} \leq 1, \quad \varepsilon \tau \leq \frac{1}{4} .
\end{aligned}
$$

It then follows from (3.37) immediately that

$$
\mathbb{E}|x(t)|^{2} \leq \frac{C_{6}}{\lambda_{1}} e^{-\varepsilon t}, \quad \forall t \geq 0 .
$$

This implies the required assertion (3.25).

Remark 3.7. As we mentioned in the introduction, [34, Theorem 4.4 ] makes a special case of our result if we take $\mu_{1}$ and $\mu_{2}$ as Dirac measures $v$ at $\delta$. Moreover, compared with condition (4.24) in [34], condition (3.24) in this theorem is more relaxed, that is to say, the time delay of our controller can be longer. In fact, with the same parameters, we can improve the result of [34, Example 5.1] to $\tau=0.0108$. 
In general, for the highly nonlinear stochastic system, the mean square exponential stability can not guarantee the almost surely exponential stability. However, in our situation, we only need to strengthen the result of Theorem 3.6, then we can get the almost surely exponential stability of the system through the $\hat{q}$ th moment stability of the system.

Theorem 3.8. Under the same Assumptions of Theorem 3.6. For any given initial data (2.9),

(i) the controlled system (3.1) is exponentially stable in $L^{\hat{q}}$ for any $\hat{q} \in[2, q)$;

(ii) the solution of the controlled system (3.1) satisfies

$$
\limsup _{t \rightarrow \infty} \frac{1}{t} \log (|x(t)|)<0 \text { a.s. }
$$

That is, the controlled system (3.1) is almost surely exponentially stable.

Proof. (i) By Theorem 3.3,

$$
C_{7}=\sup _{t \geq 0} \mathbb{E}|x(t)|^{q}<\infty .
$$

Applying the Hölder inequality, we obtain

$$
\begin{aligned}
\mathbb{E}|x(t)|^{\hat{q}} & \leq\left(\mathbb{E}|x(t)|^{2}\right)^{(q-\hat{q}) /(q-2)}\left(\mathbb{E}|x(t)|^{q}\right)^{(\hat{q}-2) /(q-2)} \\
& \leq C_{7}^{(\hat{q}-2) /(q-2)}\left(C_{6} / \lambda_{1}\right)^{(q-\hat{q}) /(q-2)} e^{-\varepsilon t(q-\hat{q}) /(q-2)}
\end{aligned}
$$

for any $\hat{q} \in[2, q)$.

(ii) By using the similar method of [37, Theorem 8.8 on p.309] and [34, Theorem 4.5], the assertion (3.39) can be obtained from conditions (2.7) and (3.40).

\section{Example}

To illustrate applications of our theory clearly, in this section, we consider the following scalar stochastic integrodifferential equation

$$
d x(t)=f\left(x_{t}, r(t), t\right) d t+g\left(x_{t}, r(t), t\right) d w(t),
$$

where the coefficients $f$ and $g$ are defined by

$$
\begin{aligned}
& f\left(x_{t}, 1, t\right)=-x(t)\left(3 x^{2}(t)+\int_{-\infty}^{0} x^{2}(t+\theta) d \mu_{1}(\theta)-1\right), \\
& g\left(x_{t}, 1, t\right)=0.5\left(-|x(t)|^{3 / 2}+\int_{-\infty}^{0}|x(t+\theta)|^{3 / 2} d \mu_{2}(\theta)\right), \\
& f\left(x_{t}, 2, t\right)=-x(t)\left(4 x^{2}(t)-\int_{-\infty}^{0} x^{2}(t+\theta) d \mu_{1}(\theta)-1.5\right), \\
& g\left(x_{t}, 2, t\right)=|x(t)|^{3 / 2}-0.5 \int_{-\infty}^{0}|x(t+\theta)|^{3 / 2} d \mu_{2}(\theta),
\end{aligned}
$$

$d \mu_{1}(\theta)=d \mu_{2}(\theta)=e^{\theta} d \theta$ on $\theta \in(-\infty, 0]$ are probability measures and $w(t)$ is a scalar Brownian motion, $r(t)$ is a Markov chain on the state space $\mathcal{S}=\{1,2\}$ with its generator

$$
\Gamma=\left(\begin{array}{cc}
-1 & 1 \\
2 & -2
\end{array}\right)
$$

This equation is widely discussed in population models (for example, [12, 13] and the reference therein). Through simple calculation, it can be found that the equation (4.1) satisfies Assumptions 2.3 and 2.4, that is, the equation (4.1) has a unique solution. Letting the initial value

$$
\xi=\left\{\begin{array}{cl}
5 e^{0.01 t}-5 e^{-1}, & \text { if } t \in(-100,0] \\
0, & \text { if } t \in(-\infty,-100]
\end{array} \quad \text { and } r(0)=1\right.
$$

The computer simulation (Figure 4.1) shows that this hybrid stochastic integro-differential equation (4.1) is not almost surely stable. 
Remark 4.1. Since the system with infinite delay is difficult to perform numerical simulation, we have chosen a special initial value (4.4) here. But this is enough to illustrate our previous theoretical results. For the theory of numerical methods of SFDEs, please refer to [38, 39, 40].
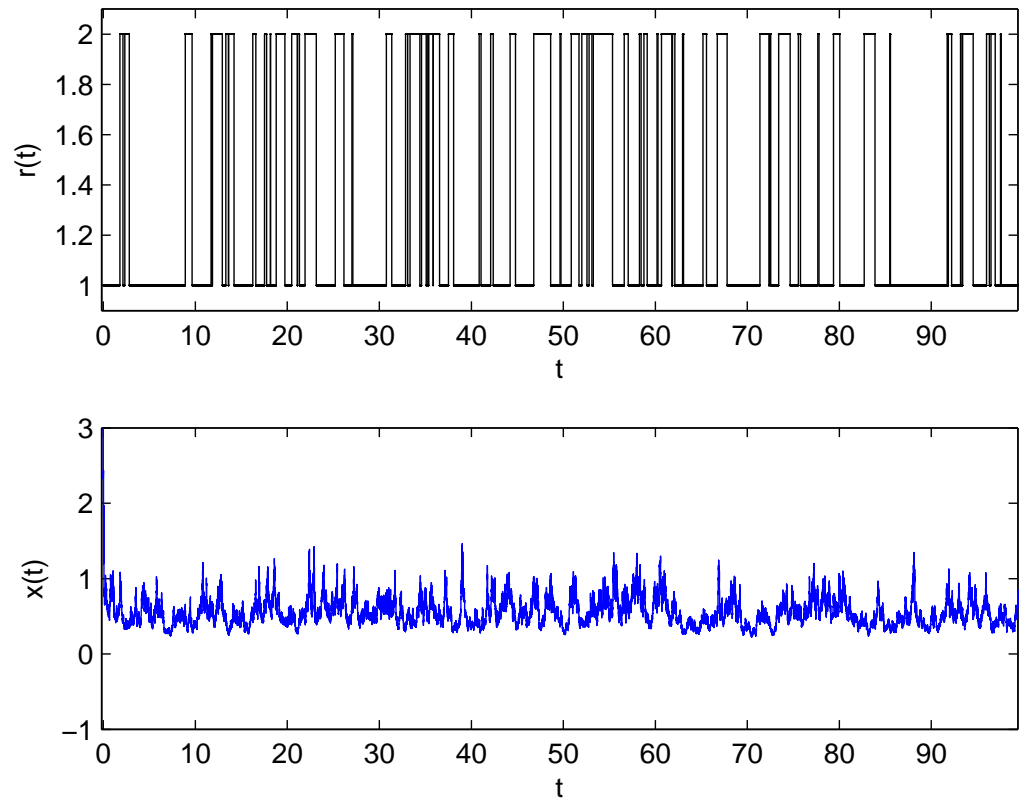

Figure 4.1: The computer simulation of the sample paths of the Markov chain and the equation (4.1) using the Euler-Maruyama method with step size $10^{-3}$.

The following describes how to design the delay feedback control to stabilize the unstable system (4.1). We will choose the control function $u: R \times \mathcal{S} \times R_{+} \rightarrow R$ define by

$$
u(x, 1, t)=-2 x, \quad u(x, 2, t)=-3 x .
$$

It is straightforward to see that Assumption 3.1 hold with $\kappa=3$. Applying Theorems 3.2 and 3.3 yield that the controlled system

$$
d x(t)=\left[f\left(x_{t}, r(t), t\right)+u(x(t-\tau), r(t), t)\right] d t+g\left(x_{t}, r(t), t\right) d w(t)
$$

has a unique global solution on $t \geq 0$ for any initial data (2.9) and the solution satisfies that

$$
\sup _{0 \leq t<\infty} \mathbb{E}|x(t, \xi)|^{q}<\infty, \quad \forall q>2 .
$$

Next, let's show that the controlled system (4.6) satisfies all of the assumptions in section 4. For $\left(x_{t}, i, t\right) \in$ $B C((-\infty, 0] ; R) \times \mathcal{S} \times R_{+}$, we have

$$
\begin{aligned}
& x(t)\left[f\left(x_{t}, i, t\right)+u(x(t), i, t)\right]+\frac{1}{2}\left|g\left(x_{t}, i, t\right)\right|^{2} \\
& \leq\left\{\begin{array}{cl}
0.0625 \int_{-\infty}^{0}|x(t+\theta)|^{2} e^{\theta} d \theta-0.9375 x^{2}(t)+0.0625 \int_{-\infty}^{0}|x(t+\theta)|^{4} e^{\theta} d \theta-2.9375 x^{4}(t), & \text { if } i=1, \\
0.0625 \int_{-\infty}^{0}|x(t+\theta)|^{2} e^{\theta} d \theta-1.25 x^{2}(t)+0.5625 \int_{-\infty}^{0}|x(t+\theta)|^{4} e^{\theta} d \theta-3.25 x^{4}(t), & \text { if } i=2,
\end{array}\right.
\end{aligned}
$$


and

$$
\begin{aligned}
& x(t)\left[f\left(x_{t}, i, t\right)+u(x(t), i, t)\right]+\frac{3}{2}\left|g\left(x_{t}, i, t\right)\right|^{2} \\
& \leq\left\{\begin{array}{cc}
0.1875 \int_{-\infty}^{0}|x(t+\theta)|^{2} e^{\theta} d \theta-0.8175 x^{2}(t)+0.1875 \int_{-\infty}^{0}|x(t+\theta)|^{4} e^{\theta} d \theta-2.8125 x^{4}(t), & \text { if } i=1, \\
0.1875 \int_{-\infty}^{0}|x(t+\theta)|^{2} e^{\theta} d \theta-0.75 x^{2}(t)+0.6875 \int_{-\infty}^{0}|x(t+\theta)|^{4} e^{\theta} d \theta-2.75 x^{4}(t), & \text { if } i=2 .
\end{array}\right.
\end{aligned}
$$

It is easy to see that

$$
\beta_{13}=-0.9375, \beta_{23}=-1.25, \hat{\beta}_{13}=-0.8125, \hat{\beta}_{23}=-0.75 \text {. }
$$

Hence,

$$
\mathcal{A}_{1}=\left(\begin{array}{cc}
2.875 & -1 \\
-2 & 4.5
\end{array}\right) \text { and } \mathcal{A}_{2}=\left(\begin{array}{cc}
4.25 & -1 \\
-2 & 5
\end{array}\right)
$$

are both M-matrices. Using (3.15), we then obtain

$$
c_{1}=0.502857, c_{2}=0.445714, \hat{c}_{1}=0.311688, \hat{c}_{2}=0.324675,
$$

and while the Assumption 3.4 is satisfied. The function $U$ defined by (3.16) becomes

$$
U(x, i)= \begin{cases}0.502857 x^{2}+0.311688 x^{4}, & \text { if } i=1, \\ 0.445714 x^{2}+0.324675 x^{4}, & \text { if } i=2 .\end{cases}
$$

Through simple calculations, combined with (3.17), we can get

$$
\mathcal{L} U\left(x_{t}, i, t\right) \leq\left\{\begin{array}{c}
0.062857 \int_{-\infty}^{0}|x(t+\theta)|^{2} e^{\theta} d \theta-x^{2}(t)+0.179740 \int_{-\infty}^{0}|x(t+\theta)|^{4} e^{\theta} d \theta \\
-3.837403 x^{4}(t)+0.155844 \int_{-\infty}^{0}|x(t+\theta)|^{6} e^{\theta} d \theta-3.428571 x^{6}(t), \\
0.055714 \int_{-\infty}^{0}|x(t+\theta)|^{2} e^{\theta} d \theta-x^{2}(t)+0.623182 \int_{-\infty}^{0}|x(t+\theta)|^{4} e^{\theta} d \theta \\
-3.775390 x^{4}(t)+0.595238 \int_{-\infty}^{0}|x(t+\theta)|^{6} e^{\theta} d \theta-3.273810 x^{6}(t),
\end{array} \quad \text { if } i=2 .\right.
$$

To verify Assumption 3.5, we let $\gamma_{1}=0.12, \gamma_{2}=0.1$ and $\gamma_{3}=1.2$. Noting

$$
\begin{gathered}
\mathcal{L} U\left(x_{t}, i, t\right)+\gamma_{1}\left(2 c_{i}|x|+4 \hat{c}_{i}|x|^{3}\right)^{2}+\gamma_{2}\left|f\left(x_{t}, i, t\right)\right|^{2}+\gamma_{3}\left|g\left(x_{t}, i, t\right)\right|^{2} \\
\leq-0.529643\left(x^{2}(t)-0.401888 \int_{-\infty}^{0}|x(t+\theta)|^{2} e^{\theta} d \theta\right) \\
-\Phi(x(t))+0.974291 \int_{-\infty}^{0} \Phi(x(t+\theta)) e^{\theta} d \theta,
\end{gathered}
$$

where $\Phi(x)=1.438081 x^{4}+0.94754 x^{6}$. That is, conditions (3.18) and (3.19) are satisfied when $\rho=0.529643, \gamma_{4}+\gamma_{5}=$ 0.401888 and $\gamma_{6}+\gamma_{7}=0.974291$. Accordingly, condition (3.24) becomes $\tau<0.017688$. By Theorem 3.8, we can therefore conclude that the controlled system (4.6) with the control function (4.3) is not only exponentially stable in $L^{\hat{q}}$ for any $\hat{q} \in[2, q)$ but also almost surely provided $\tau<0.017688$.

To perform a computer simulation, we set $\tau=0.015$ and the initial data (4.4). The sample paths of the Markov chain and the solution of the equation (4.6) are plotted in Figure 4.2. The simulation supports our theoretical results clearly.

\section{Conclusion}

In this paper we have discussed the stabilization of highly nonlinear hybrid ISFDEs by delay feedback controls. In fact, as far as the author knows, there are few results about the stability and stabilization of highly nonlinear hybrid ISFDEs. Therefore, for ISFDEs model which does not satisfy the linear growth condition, it is necessary to develop a new delay feedback stabilization theory to fill the gap. In this paper, we first construct the phase space reasonably according to the characteristics of infinite delay, and obtain the existence and uniqueness condition of the solution of hybrid system. On this basis, we design delay feedback controls, which not only make the controlled system be bounded by the $q$ th moment, but also make use of the Lyapunov functionals constructed by M-matrices to ensure the exponential stability in the sense of moment and almost surely. Finally, an example and computer simulations are given to illustrate our results. 

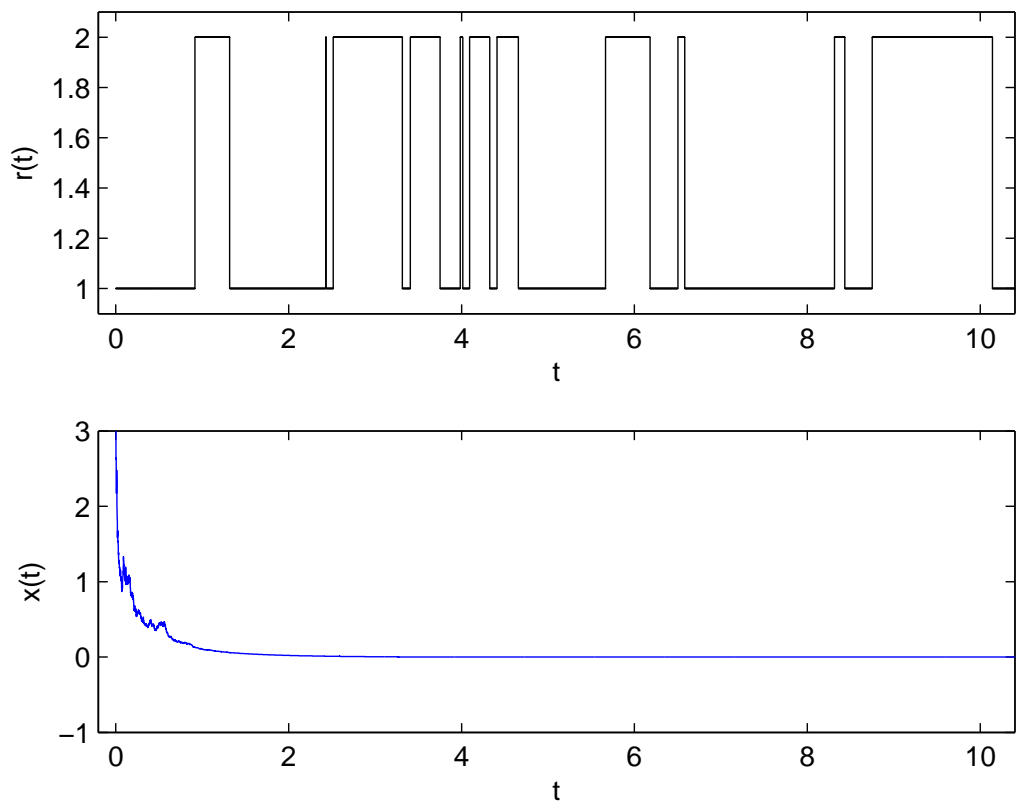

Figure 4.2: The computer simulation of the sample paths of the Markov chain and the equation (4.6) using the Euler-Maruyama method with step size $10^{-3}$.

\section{Acknowledgements}

The authors would like to thank the National Natural Science Foundation of China (71571001), the Natural Science Foundation of Universities in Anhui Province (KJ2020A0367), Startup Foundation for Introduction Talent of AHPU (2020YQQ066), the Royal Society (WM160014, Royal Society Wolfson Research Merit Award), the Royal Society and the Newton Fund (NA160317, Royal Society-Newton Advanced Fellowship), the EPSRC (EP/K503174/1) for their financial support.

\section{References}

[1] X. Mao, Stochastic Differential Equations and Applications, 2nd ed. ed., Horwood Publishing, Chichester, 2007.

[2] Q. Luo, X. Mao, Y. Shen, Generalised theory on asymptotic stability and boundedness of stochastic functional differential equations, Automatica 47 (2011) 2075-2081.

[3] J. Liu, On asymptotic convergence and boundedness of stochastic systems with time-delay, Automatica 48 (2012) 3166-3172.

[4] X. Zong, G. Yin, L. Y. Wang, T. Li, J.-F. Zhang, Stability of stochastic functional differential systems using degenerate Lyapunov functionals and applications, Automatica 91 (2018) 197-207.

[5] W. Hu, Q. Zhu, H. Karimi, Some improved Razumikhin stability criteria for impulsive stochastic delay differential systems, IEEE Transactions on Automatic Control 64 (2019) 5207-5213.

[6] F. Wei, K. Wang, The existence and uniqueness of the solution for stochastic functional differential equations with infinite delay, Journal of Mathematical Analysis and Applications 331 (2007) 516-531.

[7] Y. Ren, S. Lu, N. Xia, Remarks on the existence and uniqueness of the solutions to stochastic functional differential equations with infinite delay, Journal of Computational and Applied Mathematics 220 (2008) 364-372.

[8] X. Li, X. Fu, Stability analysis of stochastic functional differential equations with infinite delay and its application to recurrent neural networks, Journal of Computational and Applied Mathematics 234 (2010) 407-417.

[9] G. Pavlović, S. Janković, Razumikhin-type theorems on general decay stability of stochastic functional differential equations with infinite delay, Journal of Computational and Applied Mathematics 236 (2012) 1679-1690.

[10] Y. Sheng, F. L. Lewis, Z. Zeng, Exponential stabilization of fuzzy memristive neural networks with hybrid unbounded time-varying delays, IEEE Transactions on Neural Networks and Learning Systems 30 (2019) 739-750.

[11] F. Wu, Y. Xu, Stochastic Lotka-Volterra population dynamics with infinite delay, SIAM Journal on Applied Mathematics 70 (2009) $641-657$. 
[12] M. Vasilova, M. Jovanović, Stochastic Gilpin-Ayala competition model with infinite delay, Applied Mathematics and Computation 217 (2011) 4944-4959.

[13] M. Liu, D. Fan, K. Wang, Stability analysis of a stochastic logistic model with infinite delay, Communications in Nonlinear Science and Numerical Simulation 18 (2013) 2289-2294

[14] M. Liu, Global asymptotic stability of stochastic Lotka-Volterra systems with infinite delays, IMA Journal of Applied Mathematics 80 (2015) 1431-1453.

[15] M. Obradović, M. Milošević, Stability of a class of neutral stochastic differential equations with unbounded delay and Markovian switching and the Euler-Maruyama method, Journal of Computational and Applied Mathematics 309 (2017) 244-266.

[16] P. Guo, C.-J. Li, Razumikhin-type technique on stability of exact and numerical solutions for the nonlinear stochastic pantograph differential equations, BIT Numerical Mathematics 59 (2018) 77-96.

[17] Y. Hu, F. Wu, C. Huang, Robustness of exponential stability of a class of stochastic functional differential equations with infinite delay, Automatica 45 (2009) 2577-2584.

[18] F. Wu, S. Hu, Attraction, stability and robustness for stochastic functional differential equations with infinite delay, Automatica 47 (2011) 2224-2232.

[19] X. Mao, Stochastic versions of the LaSalle theorem, Journal of Differential Equations 153 (1999) 175-195.

[20] L. Hu, X. Mao, L. Zhang, Robust stability and boundedness of nonlinear hybrid stochastic differential delay equations, IEEE Transactions on Automatic Control 58 (2013) 2319-2332.

[21] L. Feng, S. Li, X. Mao, Asymptotic stability and boundedness of stochastic functional differential equations with Markovian switching, Journal of the Franklin Institute 353 (2016) 4924-4949.

[22] W. Fei, L. Hu, X. Mao, M. Shen, Delay dependent stability of highly nonlinear hybrid stochastic systems, Automatica 82 (2017) 165-170.

[23] W. Fei, L. Hu, X. Mao, M. Shen, Structured robust stability and boundedness of nonlinear hybrid delay systems, SIAM Journal on Control and Optimization 56 (2018) 2662-2689.

[24] C. Fei, M. Shen, W. Fei, X. Mao, L. Yan, Stability of highly nonlinear hybrid stochastic integro-differential delay equations, Nonlinear Analysis: Hybrid Systems 31 (2019) 180-199.

[25] Y. Kao, J. Xie, C. Wang, H. R. Karimi, A sliding mode approach to $H_{\infty}$ non-fragile observer-based control design for uncertain Markovian neutral-type stochastic systems, Automatica 52 (2015) 218-226.

[26] G. Yin, F. Wu, G. Zhao, Feedback controls to ensure global solutions and asymptotic stability of Markovian switching diffusion systems, Mathematical Control and Related Fields 5 (2015) 359-376.

[27] B. Jiang, Y. Kao, C. Gao, X. Yao, Passification of uncertain singular semi-Markovian jump systems with actuator failures via sliding mode approach, IEEE Transactions on Automatic Control 62 (2017) 4138-4143.

[28] J. Xie, Y. Kao, J. H. Park, $H_{\infty}$ performance for neutral-type Markovian switching systems with general uncertain transition rates via sliding mode control method, Nonlinear Analysis: Hybrid Systems 27 (2018) 416-436.

[29] X. Wu, Y. Tang, J. Cao, Input-to-state stability of time-varying switched systems with time delays, IEEE Transactions on Automatic Control 64 (2019) 2537-2544

[30] S. Luo, F. Deng, Stabilization of hybrid stochastic systems in the presence of asynchronous switching and input delay, Nonlinear Analysis: Hybrid Systems 32 (2019) 254-266.

[31] C. Fei, W. Fei, X. Mao, D. Xia, L. Yan, Stabilization of highly nonlinear hybrid systems by feedback control based on discrete-time state observations, IEEE Transactions on Automatic Control 65 (2020) 2899-2912.

[32] X. Mao, J. Lam, L. Huang, Stabilisation of hybrid stochastic differential equations by delay feedback control, Systems \& Control Letters 57 (2008) 927-935.

[33] Z. Lu, J. Hu, X. Mao, Stabilisation by delay feedback control for highly nonlinear hybrid stochastic differential equations, Discrete \& Continuous Dynamical Systems-B 24 (2019) 4099-4116.

[34] X. Li, X. Mao, Stabilisation of highly nonlinear hybrid stochastic differential delay equations by delay feedback control, Automatica 112 (2020) 108657.

[35] O. Kallenberg, Foundations of Modern Probability, Springer-Verlag, New York, 2006.

[36] X. Mao, Exponential Stability of Stochastic Differential Equations, Marcel Dekker, New York, 1994.

[37] X. Mao, C. Yuan, Stochastic Differential Equations with Markovian Switching, Imperial college press, London, 2006.

[38] W. Zhang, M. H. Song, M. Z. Liu, Strong convergence of the truncated Euler-Maruyama method for stochastic functional differential equations, International Journal of Computer Mathematics 95 (2017) 2363-2387.

[39] L. Liu, F. Deng, T. Hou, Almost sure exponential stability of implicit numerical solution for stochastic functional differential equation with extended polynomial growth condition, Applied Mathematics and Computation 330 (2018) 201-212.

[40] X. Li, X. Mao, G. Yin, Explicit numerical approximations for stochastic differential equations in finite and infinite horizons: truncation methods, convergence in $p$ th moment and stability, IMA Journal of Numerical Analysis 39 (2019) 847-892. 